\title{
A Flat Four-Beam Switched Array Antenna
}

\author{
Nobuhiro Kuga, Student Member, IEEE, and Hiroyuki Arai, Member, IEEE
}

\begin{abstract}
A low-profile four-beam switched array antenna for mobile terminals is proposed in this paper. A flat antenna concept modeled as a square magnetic line current array is realized by thin trapezoidal cavity elements. A unidirectional beam is obtained which can be redirected by alternating input terminals of the hybrid coupler. Element characteristics are calculated approximately using a cavity model with the two-dimensional finite-element method. Input characteristics and radiation pattern of the array antenna are evaluated by experiments.
\end{abstract}

\section{INTRODUCTION}

$\mathbf{T}$ HE problems in mobile telephone systems are multipath fading, co-channel interference and near-far problems, which will be more serious in high-speed digital communication systems in the near future. The multipath fading is caused by the several different path lengths with different arrival angles. To increase the channel capacity in a cellular system, the co-channel frequency is reused in other cells located a few cells away. However, undesired signal paths with cochannel frequency from other base stations or mobile terminals disturb a linked path and, therefore, produce the co-channel interference. On the other hand, the near-far problem in a spread spectrum system is caused by a strong signals emitted from nearby mobile terminals. To reduce the interference, antenna pattern diversity is effective.

Ikegami et al., proposed a switched-beam antenna for the antenna pattern diversity [1], and indicated that a four-beam antenna was best from the practical viewpoint. In their experiments the antenna consisted of four monopoles and reflectors on a ground plane. This is not suitable for mobile terminals because a low-profile antenna is required. A flat array antenna concept modeled as square magnetic line current array and hybrid couplers was proposed by Arai et al. [2], however, no antenna elements to satisfy the requirements had been obtained.

A flat four-beam antenna could also be a candidate for remote terminal antennas in indoor wireless local area network (LAN) systems. Requirements for the current system are more than $0 \mathrm{~dB}_{i}$ antenna gain in the horizontal plane, and $26 \mathrm{MHz}$ frequency bandwidth at $2.4 \mathrm{GHz}$ band. Therefore, the fourbeam antenna with $3 \mathrm{~dB}_{d}$ directive gain may be used with reduction of the feeding power.

In this paper, a low-profile antenna element that may be modeled as an ideal magnetic current array and a flat fourbeam switched antenna are presented. The characteristics of the antenna element are analyzed using a cavity resonator

Manuscript received February 6, 1995; revised November 6, 1995.

The authors are with the Division of Electrical and Computer Engineering, Faculty of Engineering, Yokohama National University, 156 Tokiwadai, Hodogaya-ku, Yokohama-shi, 240 Japan.

Publisher Item Identifier S 0018-926X(96)06996-7. model and the finite-element method (FEM), and are verified by experiments.

\section{ANTENNA CONFIGURATION}

A square array with four magnetic line currents is shown in Fig. 1. Opposite elements such as $A^{\prime}-B^{\prime}$ (or $C^{\prime}-D^{\prime}$ ) are fed through a $3-\mathrm{dB}$ hybrid coupler, and each element is driven in equal amplitude with phase difference of $90^{\circ}$. The cardioid radiation pattern in the $y z$ plane can be redirected to the opposite direction by alternating input terminal one and two of the hybrid coupler when the element spacing is a quarter wavelength. Beam direction of the array $C^{\prime}-D^{\prime}$ is also switched by the terminal three and four in the $z x$-plane. Then the radiation pattern of this four-element array is switched to form four beams in the $x y$ plane, as shown in Fig. 1(c). The $360^{\circ}$ azimuth coverage is obtained by switching input terminal of the hybrid coupler through terminals one to four.

Next, we propose a flat antenna, as shown in Fig. 2. It consists of a trapezoidal conducting cavity with an aperture, where we may assume a magnetic line current on its aperture for the antenna radiation. It is mounted on a ground plane and fed by a probe. The cavity thickness $h$ is one twentieth of wavelength $(0.05 \lambda)$, and aperture width $2 L$ and the back wall width $2 L_{0}$ are $0.275 \lambda$ and $1.55 \lambda$, respectively. The element may be filled with dielectric materials for miniaturizing the antenna dimension. In this paper, we will discuss an air-loaded antenna.

A flat four-beam switched antenna using this element (shown in Fig. 3) gives the ideal magnetic line current array. Each element is connected to the hybrid coupler. However, only two of the elements are excited simultaneously. Beam switching is carried out by switching input terminals of the hybrid couplers through terminal one to four. Switching timing is determined by received signal strength or error-bit rate.

\section{ANALYSIS}

Far-field pattern of the element antenna is derived using a magnetic line current $\mathbf{I}_{m}$ as

$$
\mathbf{I}_{m}=I_{m 0} \frac{\sin k_{0}(L-|x|)}{\sin k_{0} L} \mathbf{a}_{x}
$$

and the far-field pattern is given by (3)

$$
\mathbf{E}=-j \frac{I_{m 0} h}{2 \pi r \sin k_{0} L} e^{-j k_{0} r} \frac{\left[\cos \left(k_{0} L \cos \varphi\right)-\cos k_{0} L\right]}{\sin \varphi} \mathbf{a}_{\theta^{\prime}}
$$

A switched-beam pattern is derived from (1) and (2) with array factor as follows:

$$
D_{\theta}(\theta)=e^{j \delta_{1}}-e^{j \frac{\pi}{2} \sin \theta} e^{j \delta_{2}}
$$




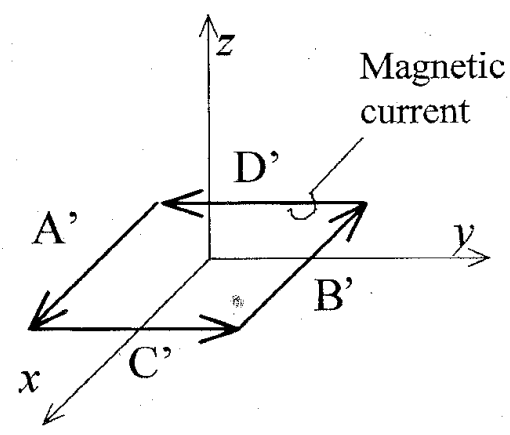

(a)

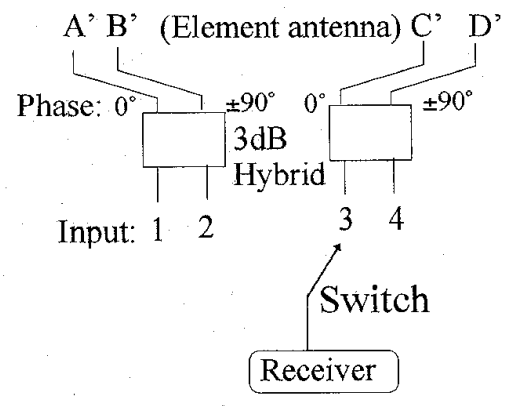

(b)

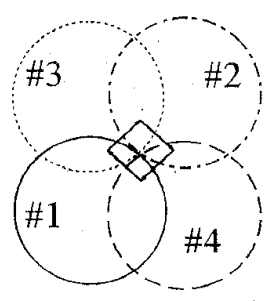

(c)

Fig. 1. Flat antenna concept for the four-beam switched antenna.

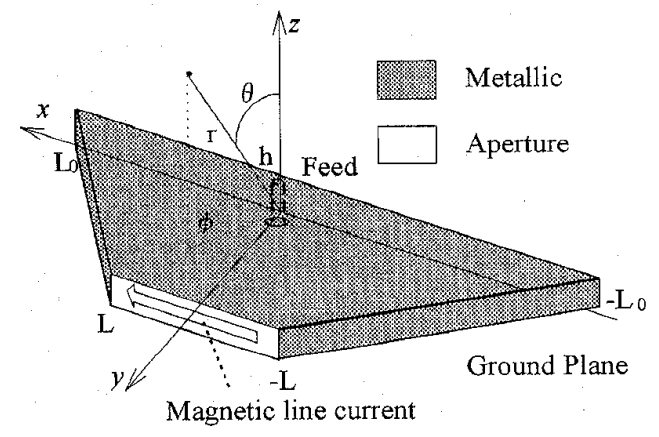

$$
2 L=0.275 \lambda, 2 L_{0}=1.55 \lambda, h=0.05 \lambda
$$

Fig. 2. Flat antenna element.

$$
\begin{array}{r}
D_{\theta}(\varphi)=\left(e^{j \delta_{1}}-e^{j \frac{\pi}{2} \sin \varphi} e^{j \delta_{2}}\right) \frac{\cos \left(k_{0} L \cos \varphi\right)-\cos k_{0} L}{\sin \varphi} \\
\left(\delta_{1}, \delta_{2}\right)=\left(0, \frac{\pi}{2}\right),\left(\frac{\pi}{2}, 0\right)
\end{array}
$$

where $k_{0}$ is wave number in free space, and $\delta_{1}, \delta_{2}$ are the initial feeding phases of the elements.

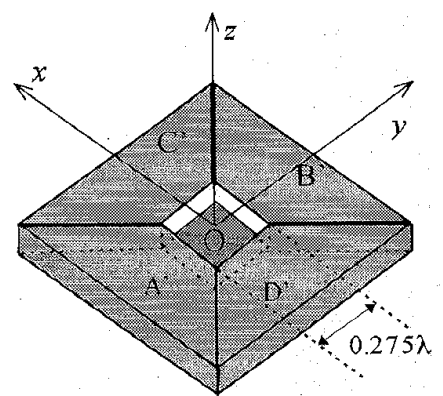

Fig. 3. Flat four-beam switched array antenna. Metallic portions shaded with gray.

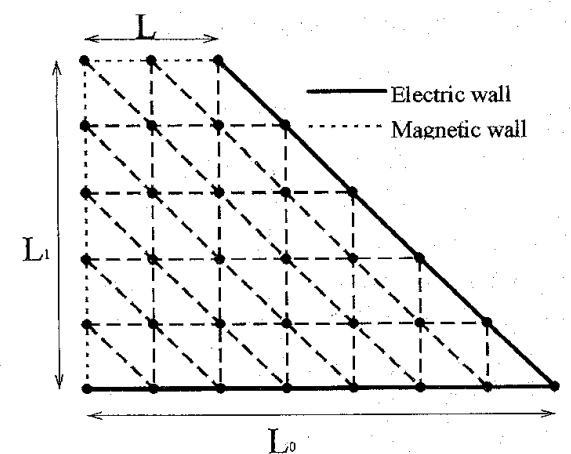

Fig. 4. Analysis model for the finite-element method.

We may assume a magnetic wall boundary on the aperture of the trapezoidal cavity because the antenna thickness is much less than the wavelength. The effect of the feeding probe is neglected for simplicity. In this cavity model, the fields inside the cavity are expressed as a transverse magnetic (TM) mode neglecting the field variation in the $z$ axis as follows:

$$
\begin{aligned}
& \mathbf{E}=\phi(x, y) \mathbf{a}_{z} \\
& \mathbf{H}=\frac{j \omega \varepsilon_{0}}{k_{c}^{2}} \mathbf{a}_{z} \times \nabla_{t} \phi=\frac{j \omega \varepsilon_{0}}{k_{c}^{2}}\left[\frac{\partial \phi}{\partial y} \mathbf{a}_{x}-\frac{\partial \phi}{\partial x} \mathbf{a}_{y}\right]
\end{aligned}
$$

where $\mathbf{a}_{x}, \mathbf{a}_{y}$, and $\mathbf{a}_{z}$ represent the unit vectors in the $x, y$, and $z$ axis. The electromagnetic fields are expressed by a potential $\phi$ which is evaluated by the finite-element method (FEM) [4]. Assuming no field variations in the $z$ axis reduces the analysis model to a two-dimensional problem. Functionals $L(\phi)$ are evaluated by the stored energy inside the cavity as

$$
L(\phi)=\iint_{\text {cavity }}\left[\left(\frac{\partial \phi}{\partial x}\right)^{2}+\left(\frac{\partial \phi}{\partial y}\right)^{2}-k_{c}^{2} \phi^{2}\right] d x d y
$$

Resonant frequency of the element is obtained as a wave number $k_{c} L$ by solving the above equation as an eigenvalue problem taking into account the boundary condition at the cavity walls. In this paper, the potential functions are approximated by the second-order polynomial expressions. The element is divided into $N$ segments as shown in Fig. 4, in which a magnetic wall condition is defined along the symmetrical axis of the element. 


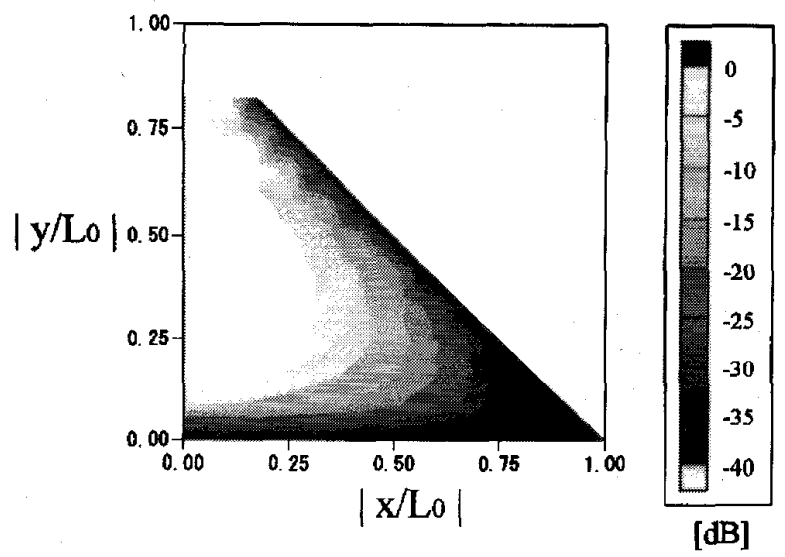

Fig. 5. Axial field ( $\phi$ ) distribution inside the cavity. The values are normalized by the maximum field strength inside the cavity.

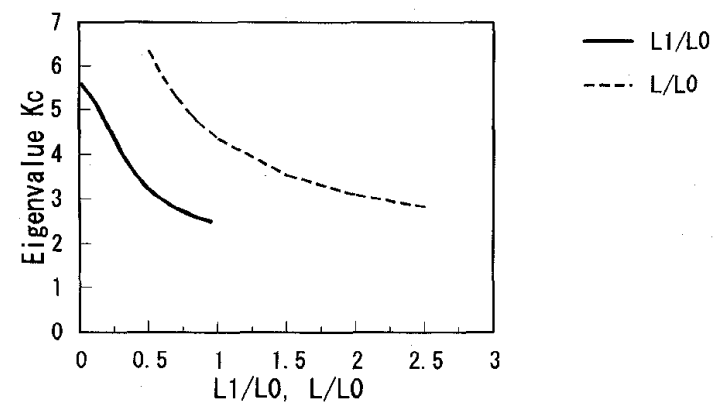

Fig. 6. Calculated eigenvalue $K_{c}$ of antenna element. The solid and broken lines keep a constant aperture width $\left(L / L_{0}=0.18\right)$ and a constant element width $\left(L_{1} / L_{0}=0.82\right)$, respectively.

\section{Characteristics OF ANTENNA}

Fig. 5 shows electric field distribution inside the antenna element at the resonant frequency. The stored energy in the element is large, and the electric field at the cavity center is larger than that near the aperture. Therefore, the unloaded quality factor of the element is high such as 60 at the height of $0.05 \lambda$. Fig. 6 shows the eigenvalue as a function of the cavity size. The eigenvalue varies on the cavity size such as $L_{1} / L_{0}$ and $L / L_{0}$.

The resonant frequency of the element antenna is determined by the cavity size, and is not only dependent on the aperture width. This is an advantage to suppress the mutual coupling between the elements. Values calculated by the FEM analysis agreed with the measurement within $2 \%$ error.

Mutual coupling less than $-20 \mathrm{~dB}$ is measured between adjacent element $A^{\prime}$ and $C^{\prime}$ because of the orthogonal location of line source elements, which may be negligible in this antenna design. Therefore, only the opposing elements $A^{\prime}$ $B^{\prime}$ will be discussed hereafter. Fig. 7 shows measured input characteristics of the subarray antenna $A^{\prime}-B^{\prime}$. The fractional frequency band width of the return loss less than $-10 \mathrm{~dB}$ is $1.0 \%$ which is wide enough for the current indoor wireless LAN system. The mutual coupling $S_{A^{\prime} B^{\prime}}$ is suppressed below $-10 \mathrm{~dB}$ and may be also negligible for antenna performance.
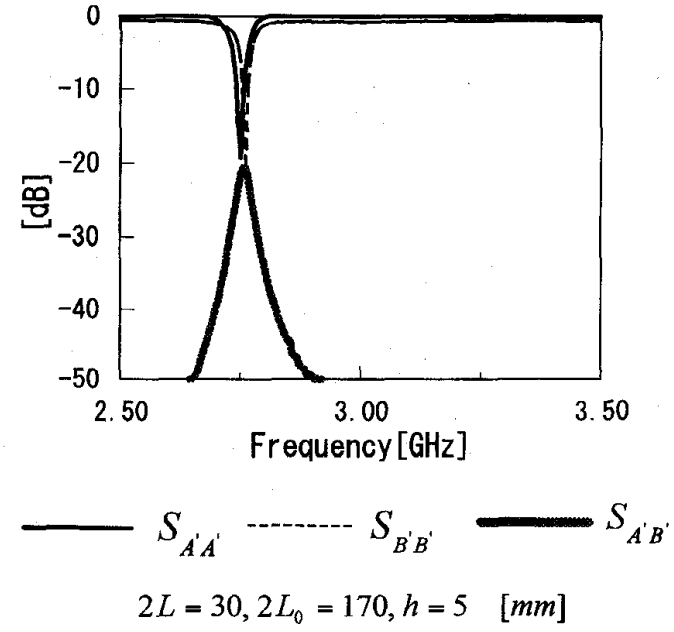

Fig. 7. Input characteristics of the subarray antenna $A^{\prime}-B^{\prime}$ without the hybrid coupler.

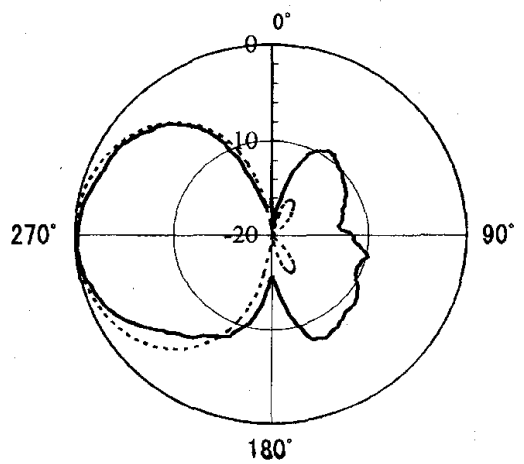

(a)

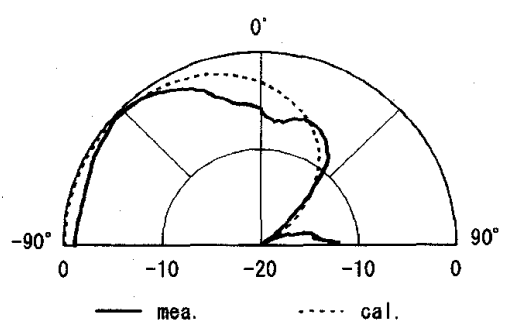

(b)

Fig. 8. Radiation pattern of the array antenna with the hybrid couplers. Port 1 of the hybrid coupler is connected to a receiver; the others $(2,3,4)$ are terminated to dummy loads.

Next, we discuss the radiation pattern of the antenna. Fig. 8 shows the vertical polarization pattern in the horizontal (xy) plane and the vertical $(y z)$ plane when Port 1 is connected to a transmitter and the other ports are terminated to dummy loads. The half-power beamwidth in the horizontal plane is $80^{\circ}$ and the directive gain is $2.7 \mathrm{~dB}_{d}$, which is suitable for four-beam switched antenna. In the vertical plane, the desired cardioide pattern is obtained, because the element spacing is about quarter wave length and mutual coupling between elements is suppressed by the resonance of the cavity. 
The pattern calculation using (1)-(4) does not agree with the experiment completely. This fact shows that the magnetic current element model is not sufficient to accurately model the radiation pattern. However, the ideal magnetic current model is applicable to the main lobe calculation of the array antenna, and is useful to predict beamwidth in the horizontal plane. Errors in the back lobe may be caused by the diffraction from the antenna edges.

\section{CONCLUSION}

We proposed a low-profile four-beam switched array antenna. The flat antenna concept using the square magnetic line current array with hybrid couplers was realized by trapezoidal cavity element with an aperture. The desired cardioide pattern was obtained in both measurement and calculation using the magnetic current model. Numerical calculation was also carried out using the cavity model analysis and the finiteelement method with two-dimensional approximations.

\section{REFERENCES}

[1] F. Ikegami, T. Takeuchi, and S. Yoshida "Characteristics of directional diversity branch in urban mobile communication," IECE Trans. (B), vol. J69-B-8, no. 8, pp. 796-803, 1986.

[2] H. Arai, H. Teramoto, and M. Toki, "Planar four-direction antenna for pattern diversity reception," IEICE Trans., vol. J73-B-11, no. 11, pp. $796-803,1990$.
[3] R. E. Collin, Antennas and Radiowave Propagation. New York: McGraw-Hill, 1991, pp. 216-265.

[4] R. C. Booton Jr., Computational Methods for Electromagnetics and Microwaves. New York: Wiley, 1992, pp. 87-111.

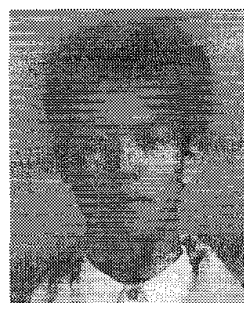

Nobuhiro Kuga (S'94) received the B.S. and M.S degrees in electrical engineering from the Yokohama National University, Japan, in 1992 and 1994, respectively. He is currently pursuing the Ph.D. degree at the Yokohama National University.

His current research interests are in the area of miniature antennas for mobile communication.

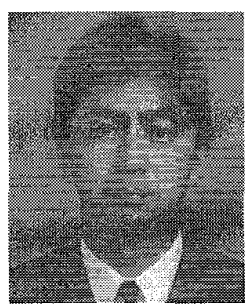

Hiroyuki Arai (S'84-M'87) received the B.S., M.S., and Ph.D. degrees in electrical engineering from the Tokyo Institute of Technology, Japan, in 1982, 1984, and 1987, respectively.

From 1987 to 1989, he was an Assistant Professor at the Tokyo Institute of Technology, Japan. He is presently an Associate Professor of electrical and computer engineering at the Yokohama National University, Japan. His current research interests include microwave circuits for RF heating and antennas and propagation in mobile communication. Dr. Arai is a member of IEICE Japan. 